\title{
Nonlinear Vibrations of a SWCNT with Geometrical Imperfection Using Nonlocal Elasticity Theory
}

\author{
Mu'tasim S. Abdel-Jaber ${ }^{1}$, Ahmad A. Al-Qaisia ${ }^{2} \&$ Nasim K. Shatarat ${ }^{1}$ \\ ${ }^{1}$ Civil Engineering Department, School of Engineering the University of Jordan, Amman, Jordan \\ ${ }^{2}$ Mechanical Engineering Department, School of Engineering the University of Jordan, Amman, Jordan \\ Correspondence: Mutasim S. Abdel-Jaber, Civil Engineering Department, School of Engineering the University \\ of Jordan, Amman 11942, Jordan. Tel: 962-6-535-5000. E-mail: m.abduljaber@ju.edu.jo
}

Received: September 10, 2017

Accepted: September 19, 2017

Online Published: September 28, 2017

doi:10.5539/mas.v11n10p91

URL: https://doi.org/10.5539/mas.v11n10p91

\begin{abstract}
The nonlinear free vibration and frequency veering of a single wall carbon nanotube (SWCNT) based on nonlocal elasticity theory is studied and investigated in this paper. The carbon nanotubes (CNT) is assumed to have an imperfection modeled as half sine and clamped at both ends. The Euler-Bernoulli Beam and Hamilton's principle were used to derive the nonlinear equation of motion of the SWCNT. The effect of; nonlocal elasticity, geometric initial rise/imperfection, and the effect of the axial force induced by mid-plane stretching are accounted for in the derivation of the nonlinear mathematical model of the CNT. The governing partial differential equation includes quadratic and cubic nonlinearities due to the initial imperfection and the mid-plane stretching. The derived equation of motion is discretized using the assumed mode method by inserting the exact linear eigen mode shape. The resulting nonlinear temporal equation was solved using the method of multiple scales (MMS) to obtain results for the nonlinear natural frequencies of the first three modes of vibrations, for different values of rise/imperfection amplitude, and for different values of the nonlocal parameter. The results are presented in non-dimensional characteristic curves to show the effect of variation of rise/imperfection amplitude and nonlocal parameter on the vibrational behavior of the CNT.
\end{abstract}

Keywords: Geometric nonlinearity, Nonlocal elasticity, Imperfection, CNT, Veering, MMS

\section{Introduction}

Since the discovery of carbon nanotubes (CNTs) in (1991) by Lijima (Lijima, 1991), they have drawn the attention of scientists/researchers and much research and studies have been devoted to analyze and understand their behavior. In addition, CNTs are gaining more interest and the nano materials are being used widely in many applications and became the core and the most important element for many applications in nanotechnology as well as nano engineering.

Their extraordinary mechanical and electrical properties made them appropriate for many applications such as; ultra-sensitive mass detection, nano-electro-mechanical systems (NEMS), radio-frequency signal processing, nano-composites as filling agents, nano-sensors, nano-actuators, nano resonators and nano-devices.

Due to the growing need, in the last two decades, to understand CNT's static and dynamic behavior; i.e. vibration, bending, buckling and post-buckling, the issue of developing accurate mathematical models for the CNTs has attracted the attention of many researchers and investigators (Eringen \& Edelen,1972; Mettler, 1962).

In the reported studies, researchers used the continuum mechanics and molecular dynamics theories to accurately study and predict the behavior of CNTs, and since the computational time from using the molecular dynamics is cumbersome, Some research and investigations were directed towards the continuum mechanics models due to their significant accuracy in modeling and predicting the dynamic and vibrational behavior of CNTs (Harik, 2002; Sazonova, Yaish, Ustunel, Rounday, Arias \& McEuen, 2004; Mayoof \& Hawwa, 2009; Hawwa \& Al-Qahtani, 2010).

Recently, studies of micro and nano beams based on the Eringen's nonlocal elasticity theory given (Eringen \& Edelen,1972), have been an active research area and many studies and investigations have been published and reported using this theory due to its importance to account for the small size/scale effect on static and dynamic behavior of micro- and nano-beams. Among these studies; 
Zhang et al. (Zhang, Liu \& Xie, 2005), studied the free transverse vibrations of double-walled carbon nanotubes. They discussed the effect of small length scale on the vibrational behavior of a double walled CNT. Additionally, they demonstrated that the natural frequencies and the associated amplitude ratios of the inner to the outer tubes are dependent upon the small length scale.

Fang et al. (Fang, Zhen, Zhang \& Tang, 2013), used the Hamilton's principle to derive the mathematical model of double-walled CNT and they took into consideration the von Karman geometric nonlinearity and van der Waals forces. The results obtained have demonstrated that the nonlocal parameter and aspect ratio affect the free nonlinear coaxial vibration of the double-walled CNT.

Mehdipour et al. (Mehdipour, Barari, Kimiaefar \& Domairry, 2102), studied the effect of curvature or waviness and mid-plane stretching on the nonlinear natural frequencies of a curved SWCNT on Pasternak elastic foundation. The amplitude frequency response curves were obtained and presented for the effect of the; amplitude of the waviness, mid-plane stretching nonlinearity, shear foundation modulus, surrounding elastic medium, radius, and length of the curved carbon nanotube on the amplitude frequency response characteristics. They concluded that the combination effects of waviness and mid-plane stretching nonlinearity on the nonlinear frequency of the curved SWCNT with a small outer radius were larger than that of a straight CNT.

(Xia \& Wang, 2010), obtained results for the vibration characteristics of fluid-conveying CNTs with curved longitudinal shape. They concluded that the curved CNTs are unconditionally stable even for a system with sufficiently high flow velocity.

Farshidianfar \& Soltani (2012), investigated the nonlinear flow-induced vibration of a SWCNT with geometrical imperfection. They observed that the nonlinear flow induced frequency ratio is decreased as the imperfection/rise of the CNT increases. Also, they studied thoroughly the effect of flow velocity, elastic foundation stiffness and boundary conditions on the reduction of natural frequency.

(Mustapha \& Zhong, 2012), studied the stability of single-walled carbon nanotubes and carbon nanocones under self-weight and axial tip force. They predicted results for the range of critical loads, and they also studied the influence of the nonlocal parameter on these critical loads.

Eltaher et al. (2012), investigated the size-dependent on static-buckling behavior of functionally graded nanobeam. They used the finite element method to study the significance of the material distribution profile, nonlocal effect, and boundary conditions on bending and buckling behavior of nanobeams.

Kong et al. (2008), used the modified couple stress theory to solve the dynamic problem of a micro beam. They presented results for the effect of size on natural frequencies for two types of micro-beams. Also, they demonstrated that the difference between the natural frequencies predicted by their model and classical beam model is very significant when the ratio of characteristic sizes to internal material length scale parameter is approximately equal to one, and diminishes when the ratio is increased.

Papanikos et al. (2008), derived expressions to evaluate the equivalent beams "geometric characteristics and elastic properties" of a CNT. They used the atomistic-based finite element in conjunction with the mechanics of material of beams that have the same tensile, bending, and torsional behavior. Also, they concluded that using a hollow cylinder is more appropriate to be used as an equivalent beam.

(Civalek \& Demir, 2011), studied the static analysis of microtubules using the method differential quadrature. They conducted numerical analysis about the effect of boundary conditions, load types, and nonlocal parameter on the static response of microtubules.

Roque et al. (2011), obtained numerical solutions to study bending, buckling, and free vibration of Timoshenko nanobeams using meshless method. The results were compared with those available in the literature. Within the same contest Thai (Thai, 2012), used nonlocal shear deformation beam theory to study bending, buckling, and vibration of nanobeams. They presented analytical solutions for deflection, buckling load, and natural frequencies and compared them to those obtained in (Roque, Ferreira \& Reddy, 2011).

Other studies were directed towards the limits of the applicability of the continuum mechanics models without any reference to the size/scale effect. Among those, (Harik, 2002) studied and analyzed thoroughly the validity of the continuum-beam models, length scale effects, and scaling analysis of the structures of CNTs. He constructed the applicability map of two classes of nanotubes; nano-beams and nano-shells. In many studies, the conditions stated in (Harik, 2002), are used to assure the validity of the continuum mechanics beam theory.

On the other hand, in some studies, researchers used the continuum mechanics models to study the dynamic and the vibrational behavior of CNTs (Ouakad \& Younis, 2011; Pantano, Boyce \& Parks, 2003). Ouakad and Younis 
(2011), studied the natural frequencies and the associated mode shapes of initially curved CNT under electric excitation. In their study, they used the Galerkin procedure to derive the nonlinear mathematical model and the discretization was based on a single mode approach using the linear mode shape of a straight beam. They presented results for the mode crossing and veering as the level of slackness/rise of a CNT and the electric excitation load are varied. As a case study, they used the dimensions of the CNT studied in (Sazonova, Yaish, Ustunel, Rounday, Arias \& McEuen, 2004).

Fathi and Hawwa (Mayoof \& Hawwa, 2009), investigated the nonlinear vibration and the chaotic behavior of a CNT with waviness along its axis. They used the continuum mechanics theory to derive the nonlinear equation of motion and they substituted a close enough shape function to obtain the nonlinear uni-modal equation of the curved CNT. They studied the effect of the mid-plane stretching and the curved geometry on the dynamic response of a CNT and the bifurcation turns to chaos.

Hawwa \& Al-Qahtani (2010), used the continuum mechanics approach to study the nonlinear oscillations and chaotic motion characteristics of a double-walled CNT. Also, they used the Galerkin's approach to discretize the coupled non-linear integro-partial differential equations of the inner and outer nano-beams. They investigated the frequency response, periodic and chaotic motions of the first and second vibration modes.

Mehdipour \& Barari (2012), studied the variation of natural frequencies of a single-walled CNT bridged with masses at different locations along the tube. They validated their results by comparing them to other mass sensor models available in the literature.

Finally, Pantano et al. (2003), used the nonlinear structural mechanics of the FE to model the structure and the deformation single-wall and multi-wall CNTs. They also studied the mechanics of wrinkling, as well as the inter-wall van der Waals forces and their effect on buckling and post-buckling behavior.

In light of the aforementioned review, one notes that a mathematical model for a CNT with initial imperfection/rise over the whole length of the CNT, using the continuum mechanics associated with the nonlocal elasticity, to the author's best knowledge, is still missing. Even for a CNT with initial rise/imperfection, to discretize the mathematical model researchers used a straight beam eigen function or used approximate eigen function, despite the fact that CNTs or beams usually exhibit a certain degree of waviness (Farshidianfar \& Soltani, 2012), which can have a significant role on the dynamic and vibrational behavior (Mehdipour, Barari, Kimiaefar \& Domairry, 2102; Farshidianfar \& Soltani, 2012; (Sazonova, Yaish, Ustunel, Rounday, Arias \& McEuen, 2004; (Hawwa \& Al-Qahtani, 2010; Mehdipour \& Barari, 2012; Mettler, 1962; Al-Qaisia \& Hamdan, $2009 ; 2010 ; 2013)$. Due to the lack of accurate mathematical models for a CNT with an arbitrary rise/imperfection using nonlocal elastic theory and continuum mechanics, this work is motivated.

The objective of this study is to derive a mathematical model based on nonlocal elasticity theory along with continuum mechanics for a CNT with geometric imperfection/initial rise and using the exact mode shape/eigen function that takes into account the waviness of the CNT to discretize the nonlinear integro-partial differential equation of motion. This study also analyzes the vibrational behavior (linear and nonlinear natural frequencies) of the CNT in which the initial imperfection is modeled as half sine wave. In addition, the objective is to evaluate the size/scale effect on the characteristic curves of nonlinear natural frequencies.

The present work intends to extend the findings of two previous studies by the author (Al-Qaisia \& Hamdan, $2009 ; 2013)$ on frequency veering by considering the effects of imperfection amplitude and size/scale, i.e. non local parameter, on the vibrational behavior of a CNT clamped at both ends.

This paper is organized as follows. In section 2 the derivation of the nonlinear mathematical model of a CNT with geometric imperfection/rise is presented followed by formulations to analyze the linear and nonlinear vibrations in sections 3 and 4, respectively. Section 5, discusses the effect of imperfection/rise amplitude and the nonlocal parameter on the CNT's dynamic and vibrational behavior. Conclusions and recommendations of this work are drawn and summarized in section 6.

\section{Derivation of the Mathematical Model of the CNT}

The carbon nanotube (CNT) under consideration is modeled as a hollow cylindrical Euler-Bernoulli beam clamped at both ends and has an initial imperfection/rise in the form of $1 / 2$ sine. The CNT is assumed to have an outside diameter $D_{o}$, inside diameter $D_{i}$, length $l$, cross sectional area $A$, area moment of inertia $I$, density $\rho$, mass per unit length $m=\rho A$ and Young's modulus $\boldsymbol{E}$.

The equation of motion of the CNT will be derived using the energy principle, i.e. by evaluating the strain/elastic and kinetic energy. To this end, based on Euler-Bernoulli beam continuum mechanics theory, the axial strain of a beam with initial rise/imperfection can be written as (Mettler, 1962). 


$$
\varepsilon_{0}=\frac{\partial u}{\partial x}+\frac{1}{2}\left(\frac{\partial w}{\partial x}\right)^{2}+\frac{\partial w_{0}}{\partial x} \frac{\partial w}{\partial x}
$$

Where $u$ and $w$ are the axial and transverse deflections, respectively. The initial imperfection/rise $w_{0}$ of the CNT is given by

$$
w_{0}=R \sin (\pi x / L)
$$

Where $R$ is the maximum amplitude of the imperfection/rise. Additional extension due to bending will have the form

$$
\varepsilon_{1}=-z \frac{\partial^{2} w}{\partial x^{2}}
$$

Where $z$ is the distance of a point material measured from the neutral axis of the beam. And the total strain is therefore

$$
\varepsilon_{x}=\varepsilon_{0}+\varepsilon_{1}
$$

The total strain and elastic energy of the beam can be expressed as

$$
U=\int_{0}^{L} \int_{A}\left(\sigma_{x} \varepsilon_{x}\right) d A d x
$$

Substituting equation (4) into equation (5), one obtains

$$
U=\int_{0}^{L} \int_{A}\left\{\sigma_{x}\left(\frac{\partial u}{\partial x}+\frac{1}{2}\left(\frac{\partial w}{\partial x}\right)^{2}+\frac{\partial w_{0}}{\partial x} \frac{\partial w}{\partial x}-z \frac{\partial^{2} w}{\partial x^{2}}\right)\right\} d A d x
$$

Where $\sigma_{x}$ is the longitudinal bending stress. Evaluating the integral given in equation (6) and imposing the rise $w_{0}$, the potential energy of the CNT can be expressed as

$$
U=\int_{0}^{L}\left\{N_{x}\left(\frac{\partial u}{\partial x}+\frac{1}{2}\left(\frac{\partial w}{\partial x}\right)^{2}+\frac{\partial w_{0}}{\partial x} \frac{\partial w}{\partial x}\right)-M_{x} \frac{\partial^{2} w}{\partial x^{2}}\right\} d x
$$

Where $N_{x}$ and $M_{x}$ are the induced normal force and bending moment at the beam's cross sectional area and are defined as;

$$
\begin{aligned}
& N_{x}=\int_{A} \sigma_{x} d A \\
& M_{x}=\int_{A} z \sigma_{x} d A
\end{aligned}
$$

The kinetic energy of the beam is given by

$$
T=\frac{\rho}{2} \int_{0}^{L} \int_{A}\left\{\left(\frac{\partial u}{\partial t}\right)^{2}+\left(\frac{\partial w}{\partial t}\right)^{2}\right\} d A d x=\frac{\rho A}{2} \int_{0}^{L}\left\{\left(\frac{\partial u}{\partial t}\right)^{2}+\left(\frac{\partial w}{\partial t}\right)^{2}\right\} d x
$$

Applying the variational methods, evaluating the Lagrangian $L=T-U=L\left(\dot{u}, \dot{w}, \dot{w}^{\prime}, u^{\prime}, w^{\prime}, w^{\prime \prime}, w\right)$ of the considered CNT and using Euler-Lagrange equation of the system Lagrangian $L$, i.e.

$$
\begin{aligned}
& \frac{\partial L}{\partial u}-\frac{\partial}{\partial t}\left(\frac{\partial L}{\partial \dot{u}}\right)-\frac{\partial}{\partial x}\left(\frac{\partial L}{\partial u^{\prime}}\right)=0 \\
& \frac{\partial L}{\partial w}-\frac{\partial}{\partial t}\left(\frac{\partial L}{\partial \dot{w}}\right)+\frac{\partial^{2}}{\partial x^{2}}\left(\frac{\partial L}{\partial w^{\prime \prime}}\right)+\frac{\partial^{2}}{\partial x \partial t}\left(\frac{\partial L}{\partial \dot{w}^{\prime}}\right)-\frac{\partial}{\partial x}\left(\frac{\partial L}{\partial w^{\prime}}\right)=0
\end{aligned}
$$

the system equations of motion for $u$ and $w$ are obtained as: 


$$
\begin{aligned}
& \rho A \frac{\partial^{2} u}{\partial t^{2}}-\frac{\partial}{\partial x}\left(N_{x}\right)=0 \\
& \rho A \frac{\partial^{2} w}{\partial t^{2}}-\frac{\partial}{\partial x}\left(N_{x}\left(\frac{\partial w}{\partial x}+\frac{\partial w_{0}}{\partial x}\right)\right)-\frac{\partial^{2} M}{\partial x^{2}}-\rho I \frac{\partial^{4} w}{\partial x^{2} \partial t^{2}}=0
\end{aligned}
$$

To include the size/scale effect in the derivation of the mathematical model of the CNT, the nonlocal Eringen's constitutive relation for the uni-axial bending stress and normal stress are (Fang, Zhen, Zhang \& Tang, 2013; 7, Eltaher, Emam \& Mahmoud, 2012)

$$
\begin{aligned}
& M_{x}-\left(e_{0} a\right)^{2} \frac{\partial^{2} M}{\partial x^{2}}=-E I w^{\prime \prime} \\
& \sigma_{x}-\left(e_{0} a\right)^{2} \frac{\partial^{2} \sigma}{\partial x^{2}}=E \varepsilon_{x}=E\left(\frac{\partial u}{\partial x}+\frac{1}{2}\left(\frac{\partial w}{\partial x}\right)^{2}+\frac{\partial w_{0}}{\partial x} \frac{\partial w}{\partial x}-z \frac{\partial^{2} w}{\partial x^{2}}\right)
\end{aligned}
$$

Where $e_{0}$ is a constant appropriate to each material and $a$ is an internal characteristic length (e.g. lattice parameter, granular distance, distance between C-C bonds) (Fang, Zhen, Zhang \& Tang, 2013).

Integrating equation (16) over the beam area $A, 0=\int z d A$, one obtains

$$
N_{x}-\left(e_{0} a\right)^{2} \frac{\partial^{2} N_{x}}{\partial x^{2}}=E A\left(\frac{\partial u}{\partial x}+\frac{1}{2}\left(\frac{\partial w}{\partial x}\right)^{2}+\frac{\partial w_{0}}{\partial x} \frac{\partial w}{\partial x}\right)
$$

Differentiate (17) with respect to $x$, we have

$$
\frac{d N_{x}}{d x}-\left(e_{0} a\right)^{2} \frac{d}{d x}\left(\frac{\partial^{2} N_{x}}{\partial x^{2}}\right)=E A\left(\frac{\partial^{2} u}{\partial x^{2}}+\frac{\partial w}{\partial x} \frac{\partial^{2} w}{\partial x^{2}}+\frac{\partial w_{0}}{\partial x} \frac{\partial^{2} w}{\partial x^{2}}+\frac{\partial w}{\partial x} \frac{\partial^{2} w_{0}}{\partial x^{2}}\right)
$$

Substituting from (13), $\rho A \frac{\partial^{2} u}{\partial t^{2}}=\frac{\partial}{\partial x}\left(N_{x}\right)$ and $\frac{\partial^{2} N_{x}}{\partial x^{2}}=\frac{\partial}{\partial x}\left(\rho A \frac{\partial^{2} u}{\partial t^{2}}\right)$ into (18), leads to

$$
\rho A \frac{\partial^{2} u}{\partial t^{2}}-\left(e_{0} a\right)^{2} \frac{\partial^{2}}{\partial x^{2}}\left(\rho A \frac{\partial^{2} u}{\partial t^{2}}\right)=E A\left(\frac{\partial^{2} u}{\partial x^{2}}+\left(\frac{\partial w}{\partial x}+\frac{\partial w_{0}}{\partial x}\right) \frac{\partial^{2} w}{\partial x^{2}}+\frac{\partial w}{\partial x} \frac{\partial^{2} w_{0}}{\partial x^{2}}\right)
$$

Solving for $\frac{\partial^{2} M_{x}}{\partial x^{2}}$ from (14) and substituting into (15), one obtains

$$
M_{x}-\left(e_{0} a\right)^{2}\left\{\rho A \frac{\partial^{2} w}{\partial t^{2}}-\frac{\partial}{\partial x}\left(N_{x}\left(\frac{\partial w}{\partial x}+\frac{\partial w_{0}}{\partial x}\right)\right)\right\}=-E I \frac{\partial^{2} w}{\partial x^{2}}
$$

Differentiate twice with respect to $x$ we can get

$$
\frac{\partial^{2} M_{x}}{\partial x^{2}}-\left(e_{0} a\right)^{2} \frac{\partial^{2}}{\partial x^{2}}\left\{\rho A \frac{\partial^{2} w}{\partial t^{2}}-\frac{\partial}{\partial x}\left(N_{x}\left(\frac{\partial w}{\partial x}+\frac{\partial w_{0}}{\partial x}\right)\right)\right\}=-E I \frac{\partial^{4} w}{\partial x^{4}}
$$

For a slender non-movable end conditions, the variation of the longitudinal inertia forces can be neglected and the axial strain $\varepsilon_{0}$ does not depend on $x$, and with the help of equation (13), it then follows that

$$
N_{x}=\frac{E A}{L}\left\{\int_{0}^{L}\left(\frac{1}{2}\left(\frac{\partial w}{\partial x}\right)^{2}+\frac{\partial w_{0}}{\partial x} \frac{\partial w}{\partial x}\right) d x\right\}
$$

Substituting the expressions of $N_{x}$ and $\partial^{2} M_{x} / \partial x^{2}$ into (21), the nonlinear integro-partial differential 
equation of motion of the nonlocal SWCNT can be derived and given as

$$
\begin{aligned}
E I \frac{\partial^{4} w}{\partial x^{4}} & +\rho A \frac{\partial^{2} w}{\partial t^{2}}-\frac{E A}{L}\left\{\int_{0}^{L}\left(\frac{1}{2}\left(\frac{\partial w}{\partial x}\right)^{2}+\frac{\partial w_{0}}{\partial x} \frac{\partial w}{\partial x}\right) d x\right\}\left(\frac{\partial^{2} w}{\partial x^{2}}+\frac{\partial^{2} w_{0}}{\partial x^{2}}\right) \\
& -\left(e_{0} a\right)^{2}\left\{-\frac{E A}{L}\left\{\int_{0}^{L}\left(\frac{1}{2}\left(\frac{\partial w}{\partial x}\right)^{2}+\frac{\partial w_{0}}{\partial x} \frac{\partial w}{\partial x}\right) d x\right\}\left(\frac{\partial^{4} w}{\partial x^{4}}+\frac{\partial^{4} w_{0}}{\partial x^{4}}\right)\right\}=0
\end{aligned}
$$

The above equation can be re-arranged and have the form

$$
\begin{aligned}
& E I \frac{\partial^{4} w}{\partial x^{4}}+\rho A\left(\frac{\partial^{2} w}{\partial t^{2}}-\left(e_{0} a\right)^{2} \frac{\partial^{4} w}{\partial x^{2} \partial t^{2}}\right)+ \\
& -\frac{E A}{L}\left\{\left\{\int_{0}^{L}\left(\frac{1}{2}\left(\frac{\partial w}{\partial x}\right)^{2}+\frac{\partial w_{0}}{\partial x} \frac{\partial w}{\partial x}\right) d x\right\}\left[\left(\frac{\partial^{2} w}{\partial x^{2}}+\frac{\partial^{2} w_{0}}{\partial x^{2}}\right)-\left(e_{0} a\right)^{2}\left(\frac{\partial^{4} w}{\partial x^{4}}+\frac{\partial^{4} w_{0}}{\partial x^{4}}\right)\right]\right\}=0
\end{aligned}
$$

The last equation represents the equation of motion of a CNT with initial imperfection/rise $w_{0}$, as given in equation (2). To simplify the analysis, the following non-dimensional parameters with respect to the cross sectional area radius of gyration $r$ of the CNT, are introduced

$$
y=w / r, \quad y_{0}=w_{0} / r, b=R / r, \quad \xi=x / L, t^{*}=t \sqrt{E I / m L^{4}}, \mu=e_{0} a / L \text { and } r=\sqrt{I / A}
$$

Where $I=\pi\left(D_{0}^{4}-D_{i}^{4}\right) / 64, A=\pi\left(D_{0}^{2}-D_{i}^{2}\right) / 4, D_{0}$ and $D_{i}$ are the outer and inner diameters of the CNT respectively.

Substituting the above dimensionless parameters into equation (24), the equation of motion of the CNT that takes into account the size effect takes the form;

$$
y^{\prime \prime \prime \prime}+\ddot{y}-\mu^{2} \ddot{y}^{\prime \prime}-\left\{\int_{0}^{1}\left(\frac{y^{\prime 2}}{2}+y^{\prime} y_{0}\right) d \xi\left\{\left(y^{\prime \prime}+y_{0}^{\prime \prime}\right)-\mu^{2}\left(y_{0}^{\prime \prime \prime}+y^{\prime \prime \prime}\right)\right\}\right\}=0
$$

Where dots are derivatives with respect to $t^{*}$, and primes derivative with respect to $\xi$. As mentioned previously, in the present study, the initial rise/imperfection $y_{0}$ is assumed to be a half sinusoid and given by

$$
y_{0}=b \sin (\pi \xi)
$$

Where $b$ is the non-dimensional rise amplitude equal to the actual rise amplitude $R$ divided by the CNT's radius of gyration $r$, i.e. $b=R / r$.

\section{Linear Vibration Analysis (Natural Frequencies and Frequency Veering)}

It was reported in previous studies (Ouakad \& Younis, 2011; Al-Qaisia \& Hamdan, 2010; 2009; 2013) that the veering phenomenon takes place for structures such as the CNT under consideration. This phenomenon occurs when the amplitude imperfection/initial rise is varied as the two frequencies approach each other causing them to veer away from one another. Also, this phenomenon is always associated with a drastic change in mode shapes and sometimes leads to mode localization, snap through, period doubling bifurcations and chaos. This phenomenon, i.e. frequency veering, can be explored from analyzing the natural frequencies obtained from the linearized version of the nonlinear integral-partial differential equation (25), which takes the form

$$
\left.y^{\prime \prime \prime \prime}+\ddot{y}-\mu^{2} \ddot{y}^{\prime \prime}-\left\{\int_{0}^{1}\left(y^{\prime} y_{0}\right) d \xi\right\}\left(y_{0}^{\prime \prime}-\mu^{2} y_{0}^{\prime \prime \prime}\right)\right\}=0
$$

To discretize the linearized version of the partial differential equation, here in this study, the single mode Galerkin's approach will be used by assuming $y=\phi(\xi) q\left(t^{*}\right)=\phi(\xi) A_{m} \sin \left(\omega_{L} t^{*}\right)$, i.e. harmonic in time, where $\omega_{L}$ is the unknown linear natural frequency. Substituting for $y$ and $y_{0}$ leads to the following boundary value problem: 


$$
\phi^{\prime \prime \prime \prime}-\omega_{L}^{2}\left(\phi-\mu^{2} \phi^{\prime \prime}\right)+=\left\{\left(-\mu^{2} \pi^{4}-\pi^{2}\right)\left(b^{2} \pi\right) \int_{0}^{l}\left(\phi^{\prime} \cos (\pi \xi)\right) d \xi\right\} \sin (\pi \xi)
$$

It is clear that for a beam without imperfection/rise, i.e. $b=0$, the right side of equation (28) is zero and thus this equation has only a homogenous solution and represents the equation of motion of a straight beam. Also, for $\mu=0$, the equation reduces to the classical Euler-Bernoulli beam obtained from the classical continuum mechanics model, i.e. local elasticity theory. However, for the CNT with imperfection/rise, the total solution $\phi(\xi)$ of the non-homogeneous linear boundary value ordinary differential equation (28) will include a particular part, i.e. a solution of the non-homogeneous linear boundary value ordinary differential equation (28) is given by $\phi(\xi)=\phi_{h}(\xi)+\phi_{p}(\xi)$, where $\phi_{h}(\xi)$ is the homogenous part and $\phi_{p}(\xi)$ is the particular solution. The homogeneous solution, after substituting $\phi_{h}(\xi)=e^{s \xi}$, is given by

$$
\phi_{h}(\xi)=A_{1} \sin \theta \xi+A_{2} \cos \theta \xi+A_{3} \sinh \gamma \xi+A_{4} \cosh \gamma \xi
$$

where

$$
\theta=\sqrt{\frac{\mu^{2} \alpha^{4}}{2}+\sqrt{\frac{\mu^{4} \alpha^{8}}{4}+\alpha^{4}}} \quad \gamma=\sqrt{\frac{-\mu^{2} \alpha^{4}}{2}+\sqrt{\frac{\mu^{4} \alpha^{8}}{4}+\alpha^{4}}} \quad \text { and } \quad \alpha=\omega_{L}^{2} .
$$

The arbitrary constants $A_{1}, A_{2}, A_{3}$ and $A_{4}$, can be determined from the following boundary conditions for clamped-clamped end conditions:

$$
\phi(0)=\phi^{\prime}(0)=\phi(1)=\phi^{\prime}(1)=0
$$

The particular solution $\phi_{p}(\xi)$ of equation (28), takes the form

$$
\phi_{p}(\xi)=D \sin (\pi \xi)
$$

Where $D$ is a constant obtained by substituting equation (31) into equation (28) whereby one obtains

$$
D=\frac{-\left[\pi^{2}+\mu^{2} \pi^{4}\right] b^{2} \pi \int_{0}^{1} \phi^{\prime} \cos (\pi \xi) d \xi}{\pi^{4}-\alpha^{4}-\mu^{2} \alpha^{4} \pi^{2}}
$$

Thus the total solution $\phi(\xi)=\phi_{h}(\xi)+\phi_{p}(\xi)$ is given by

$$
\phi(\xi)=A_{1} \sin \theta \xi+A_{2} \cos \theta \xi+A_{3} \sinh \gamma \xi+A_{4} \cosh \gamma \xi+D \sin (\pi \xi)
$$

The procedure of solving for; $A_{1}, A_{2}, A_{3}, A_{4}$ and $D$, is similar to that followed in (Al-Qaisia \& Hamdan, $2010 ; 2009 ; 2013)$. Substituting equation (33) into equation (32), one obtains

$$
D=\frac{-\left(1+\mu^{2} \pi^{2}\right) \pi^{3} b^{2}}{\pi^{4}-\left(1+\mu^{2} \pi^{2}\right) \alpha^{4}}\left\{\int_{0}^{1}\left(\begin{array}{l}
\theta A_{1} \cos \theta \xi-\theta A_{2} \sin \theta \xi+\gamma A_{3} \cosh \gamma \xi+ \\
\gamma A_{4} \sinh \gamma \xi+\pi D \cos (\pi \xi)
\end{array}\right) \cos (\pi \xi) d \xi\right\}
$$

The constant $D$ can be obtained and calculated form the following expression, after some mathematical manipulations, it takes the form

$$
D=\left(\frac{a_{5}}{a_{d}} A_{1}+\frac{a_{6}}{a_{d}} A_{2}+\frac{a_{7}}{a_{d}} A_{3}+\frac{a_{8}}{a_{d}} A_{4}\right)
$$

where 


$$
\begin{aligned}
& a_{5}=\frac{-\left(1+\mu^{2} \pi^{2}\right) \pi^{3} b^{2}}{\pi^{4}-\left(1+\mu^{2} \pi^{2}\right) \alpha^{4}} \theta\left\{\int_{0}^{1} \cos \theta \xi \cos (\pi \xi) d \xi\right\} \\
& a_{6}=\frac{\left(1+\mu^{2} \pi^{2}\right) \pi^{3} b^{2}}{\pi^{4}-\left(1+\mu^{2} \pi^{2}\right) \alpha^{4}} \theta\left\{\int_{0}^{1} \sin \theta \xi \cos (\pi \xi) d \xi\right\} \\
& a_{7}=\frac{-\left(1+\mu^{2} \pi^{2}\right) \pi^{3} b^{2}}{\pi^{4}-\left(1+\mu^{2} \pi^{2}\right) \alpha^{4}} \gamma\left\{\int_{0}^{1} \cosh \gamma \xi \cos (\pi \xi) d \xi\right\} \\
& a_{8}=\frac{-\left(1+\mu^{2} \pi^{2}\right) \pi^{3} b^{2}}{\pi^{4}-\left(1+\mu^{2} \pi^{2}\right) \alpha^{4}} \gamma\left\{\int_{0}^{1} \sinh \gamma \xi \cos (\pi \xi) d \xi\right\} \\
& a_{d}=\frac{-\left(1+\mu^{2} \pi^{2}\right) \pi^{4} b^{2}}{\pi^{4}-\left(1+\mu^{2} \pi^{2}\right) \alpha^{4}}\left\{\int_{0}^{1}[\cos (\pi \xi)]^{2} d \xi\right\}
\end{aligned}
$$

At this stage, the constant $D$ can be obtained in terms of $A_{1}, A_{2}, A_{3}$ and $A_{4}$, as given in equation (35). Then the total solution, given in (33), can be obtained and calculated by applying the four boundary conditions given in (30), which yields a system of linear equations for the arbitrary constants; $A_{1}, A_{2}, A_{3}$ and $A_{4}$. The obtained equations can be written in a matrix form, i.e. $4 \times 4$ matrix equation, where the SWCNT linear natural frequency $\omega_{L}$, for a given value of initial imperfection/rise $b$ and nonlocal parameter $\mu=e_{0} a / l$, can be obtained by setting the determinant of the coefficient matrix to zero. Once the linear natural frequency is calculated, the corresponding mode shape can be obtained from equations (33) and (35).

It is worth mentioning that the obtained mode shape is an exact one which takes into consideration the waviness of the CNT, i.e. the assumed $1 / 2$ sine initial imperfection/rise.

\section{Nonlinear Vibration Analysis}

The nonlinear integral-partial differential equation (25), can be discretized by assuming

$$
y\left(\xi, t^{*}\right)=\sum_{i=1,2}^{\infty} \phi_{i}(\xi) q_{i}\left(t^{*}\right)
$$

where $\phi_{i}(\xi)$ and $q_{i}\left(t^{*}\right)$ are the associated linear mode shape of the beam and the generalized coordinates, respectively. Here in this study, the associated linear mode shape $\phi_{i}(\xi)$ is the exact mode shape obtained in the previous section, unlike other studies in which the mode shape of the straight beam, or an approximate one, were used (Ouakad \& Younis, 2011; Mayoof \& Hawwa, 2009; Mehdipour \& Barari, 2012). Also, it was shown in previous studies (Al-Qaisia \& Hamdan, 2010; 2009; 2013), that using the single mode approach is enough to capture the dynamics of the CNT beam.

Using the single mode approach, i.e. $y=\phi(\xi) q(t)$ and substituting $y_{0}$ from equation (26) into equation (25), multiplying by $\phi(\xi)$, integrating from 0 to 1 , and for convenience using the abbreviations $\phi$ and $q$ instead of $\phi(\xi)$ and $q\left(t^{*}\right)$, respectively, one obtains the following reduced single-mode nonlinear temporal equation:

$$
\begin{aligned}
& \left\{\int_{0}^{1}\left(\phi^{2}-\mu^{2} \phi \phi^{\prime \prime}\right) d \xi\right\} \ddot{q}+\left\{\int_{0}^{1}\left(\phi \phi^{\prime \prime \prime}\right) d \xi-\int_{0}^{l} \phi\left(\int_{0}^{1} \phi^{\prime} y_{0}^{\prime} d \xi\right) y_{0}^{\prime \prime} d \xi+\mu^{2} \int_{0}^{l} \phi\left(\int_{0}^{1} \phi^{\prime} y_{0}^{\prime} d \xi\right) y_{0}^{\prime \prime \prime} d \xi\right\} q \\
& +\left\{\mu^{2} \int_{0}^{1} \phi\left(\int_{0}^{1} \frac{\phi^{\prime}}{2} d \xi\right) y_{0}^{\prime \prime \prime} d \xi+\mu^{2} \int_{0}^{l} \phi\left(\int_{0}^{1} \phi^{\prime} y_{0}^{\prime} d \xi\right) \phi^{\prime \prime \prime} d \xi-\int_{0}^{l} \phi\left(\int_{0}^{1} \frac{\phi^{\prime}}{2} d \xi\right) y_{0}^{\prime \prime} d \xi-\int_{0}^{1} \phi\left(\int_{0}^{1} \phi^{\prime} y_{0}^{\prime} d \xi\right) \phi^{\prime \prime}\right\} q^{2} \\
& +\left\{\mu^{2} \int_{0}^{1} \phi\left(\int_{0}^{1} \frac{\phi^{\prime 2}}{2} d \xi\right) \phi^{\prime \prime \prime} d \xi-\int_{0}^{1} \phi\left(\int_{0}^{1} \frac{\phi^{\prime 2}}{2} d \xi\right) \phi^{\prime \prime} d \xi\right\} q^{3}=0
\end{aligned}
$$


Equation (40) can be re-arranged and written in the from

$$
\beta_{0} \ddot{q}+\beta_{1} q+\beta_{2} q^{2}+\beta_{3} q^{3}=0
$$

Where

$$
\begin{gathered}
\beta_{0}=\int_{0}^{l}\left(\phi^{2}-\mu^{2} \phi \phi^{\prime \prime}\right) d \xi \\
\beta_{1}=\int_{0}^{l}\left(\phi \phi^{\prime \prime \prime \prime}\right) d \xi-\int_{0}^{1} \phi\left(\int_{0}^{1} \phi^{\prime} y_{0}^{\prime} d \xi\right) y_{0}^{\prime \prime} d \xi+\mu^{2} \int_{0}^{l} \phi\left(\int_{0}^{1} \phi^{\prime} y_{0}^{\prime} d \xi\right) y_{0}^{\prime \prime \prime} d \xi \\
\beta_{2}=\mu^{2} \int_{0}^{1} \phi\left(\int_{0}^{1} \frac{\phi^{\prime}}{2} d \xi\right) y_{0}^{\prime \prime \prime \prime} d \xi+\mu^{2} \int_{0}^{1} \phi\left(\int_{0}^{1} \phi^{\prime} y_{0}^{\prime} d \xi\right) \phi^{\prime \prime \prime \prime} d \xi-\int_{0}^{1} \phi\left(\int_{0}^{1} \frac{\phi^{\prime}}{2} d \xi\right) y_{0}^{\prime \prime} d \xi-\int_{0}^{1} \phi\left(\int_{0}^{1} \phi^{\prime} y_{0}^{\prime} d \xi\right) \phi^{\prime \prime} \\
\beta_{3}=\mu^{2} \int_{0}^{l} \phi\left(\int_{0}^{1} \frac{\phi^{\prime 2}}{2} d \xi\right) \phi^{\prime \prime \prime} d \xi-\int_{0}^{1} \phi\left(\int_{0}^{1} \frac{\phi^{\prime 2}}{2} d \xi\right) \phi^{\prime \prime} d \xi
\end{gathered}
$$

The expressions of $\beta_{i}$ given in equations (42-45) contain all of the CNT physical and geometrical parameters: size/scale effect $\mu=e_{0} a / l$ and initial imperfection/rise amplitude $b=R / r$. The calculation of $\beta_{i}$ for a given value or combination of the CNT parameters were carried out numerically using a developed code on MATLAB ${ }^{\circledR}$ Software. The non-dimensional natural frequency $\omega$ can also be calculated from equation (41), i.e. $\omega_{L}=\sqrt{\beta_{1} / \beta_{0}}$, which is equal to the one calculated in the previous section from the determinant of the coefficient matrix.

For convenience, equation (45) can be scaled and rewritten in the following non-dimensional form:

$$
\ddot{q}+q+\varepsilon_{2} q^{2}+\varepsilon_{3} q^{3}=0
$$

where a dot denotes a derivative with respect to $T=\left(\beta_{1} / \beta_{0}\right)^{1 / 2} t^{*}=\omega t^{*}$, and $\varepsilon_{2}$ and $\varepsilon_{3}$ are dimensionless coefficients defined as $\varepsilon_{2}=\beta_{2} / \beta_{1}$ and $\varepsilon_{3}=\beta_{3} / \beta_{1}$. As it can be concluded from the non-dimensional equation (46), the calculated nonlinear natural frequency will be equal to 1 , if the nonlinear parts are neglected. Accordingly, the non-dimensional nonlinear natural frequency that will be calculated from the non-dimensional equation (46), will be a non-dimensional ratio that relates the nonlinear frequency to the linear one, i.e. $\omega_{N L} / \omega_{L}$.

The coefficient of the quadratic nonlinearity $\varepsilon_{2}$ is due to the initial rise/imperfection $b=R / r$ while the coefficient of the cubic nonlinearity $\varepsilon_{3}$ is due to the mid-plane stretching. Both coefficients are functions of all of the CNT physical and geometrical parameters and include the size/scale effect, i.e. nonlocal parameter $\mu=e_{0} a / l$.

In this study, the non-dimensional nonlinear natural frequencies of the nonlinear oscillator given in equation (46) can be obtained from using one of the perturbation techniques such as the method of Harmonic Balance Method (HB) or the method of Multiple Scales (MMS) (Nayfeh \& Mook, 1979). It is worth mentioning that using the HB method will result in the assumed solution to contain a bias constant term since the oscillator given in equation (46) includes asymmetric nonlinearity "quadratic term $\mathrm{q}^{\wedge} 2$ ". However, more details about the HB method, both single or two terms, can be found in (Al-Qaisia \& Hamdan, 2010; 2009; 2013). Here in this paper, the nonlinear natural frequencies of the SWCNT will be obtained using the MMS method, and the expression will have the form (Al-Qaisia \& Hamdan, 2009; Nayfeh \& Mook, 1979);

$$
\left(\omega_{N L} / \omega_{L}\right)=1+\left(\frac{3}{8} \varepsilon_{3}-\frac{5}{12} \varepsilon_{2}^{2}\right) A^{2}
$$

Where $\omega_{N L} / \omega$, as mentioned before, is the non-dimensional ratio that relates the non-linear to linear natural frequency of the CNT, and $A$ is the amplitude of the motion. 


\section{Results and Discussion}

To simplify the numerical solutions of the dynamic behavior of the CNT, all linear and nonlinear results are presented in non-dimensional forms. Also, it worth mentioning that results for $b=0$, represents the case of

$$
\mu=0
$$

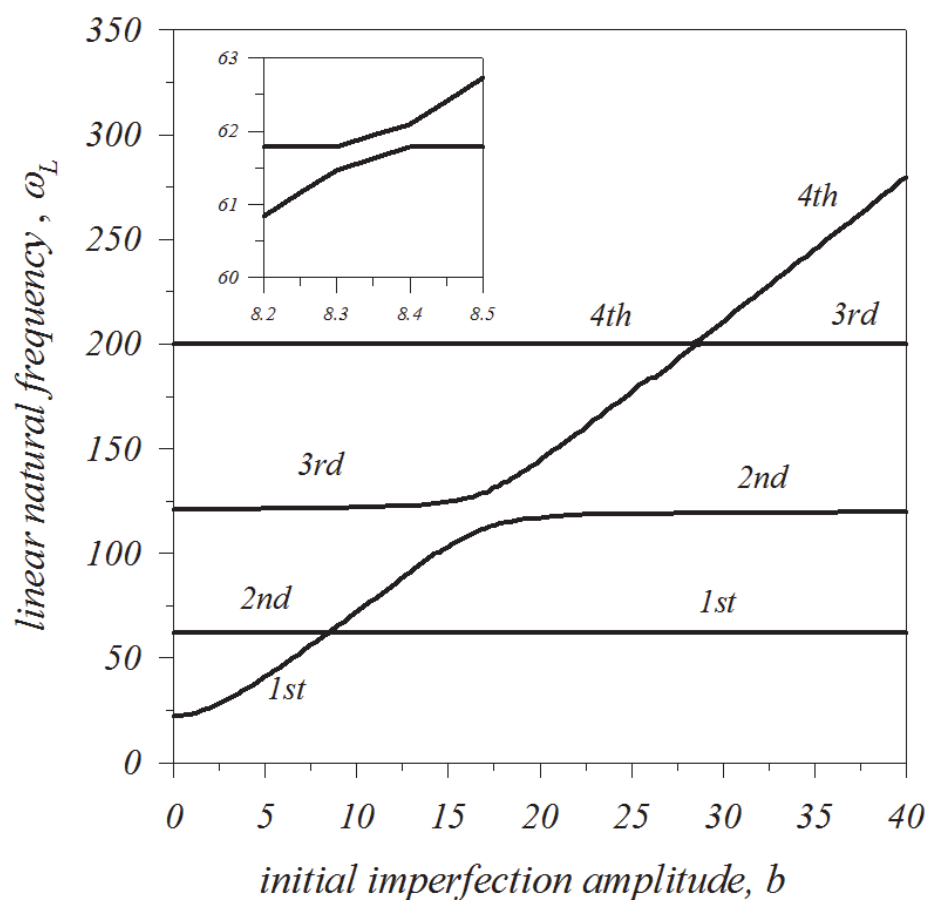

Figure 1. Variation of linear natural frequencies $\omega$ versus the CNT rise/imperfection $b$ and the zoomed view of the first veering zone, for the classical theory $\mu=0$

$$
\mu=0.2
$$

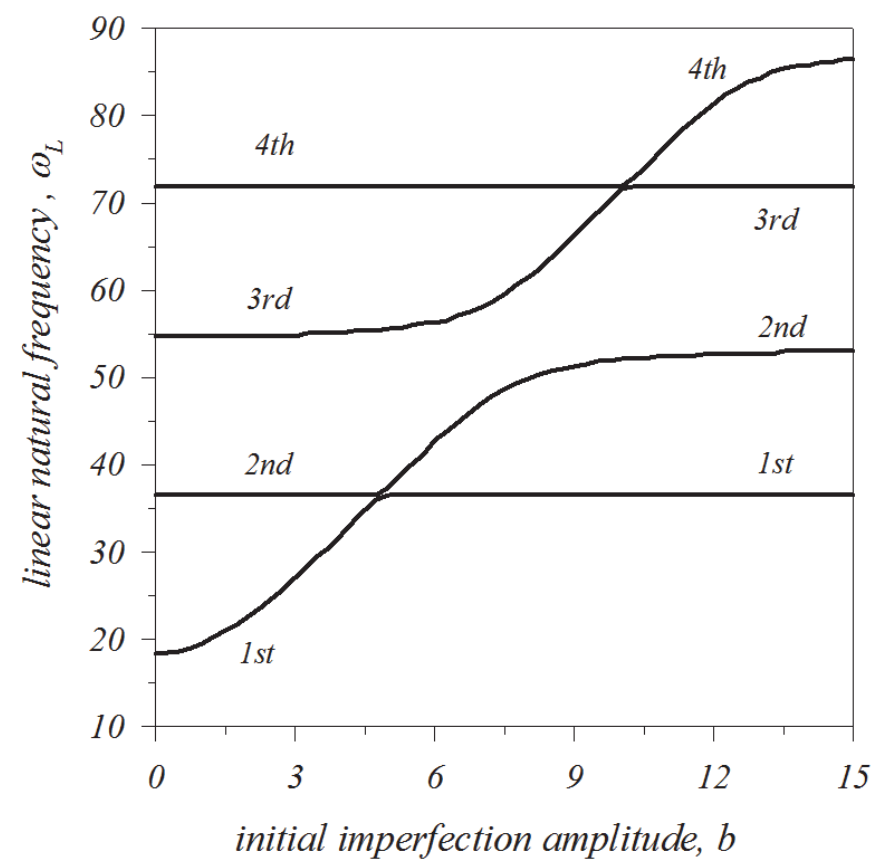

Figure 2. Variation of linear natural frequencies $\omega$ versus the CNT rise/imperfection $b$ for the case $\mu=0.2$ 


$$
b=0
$$

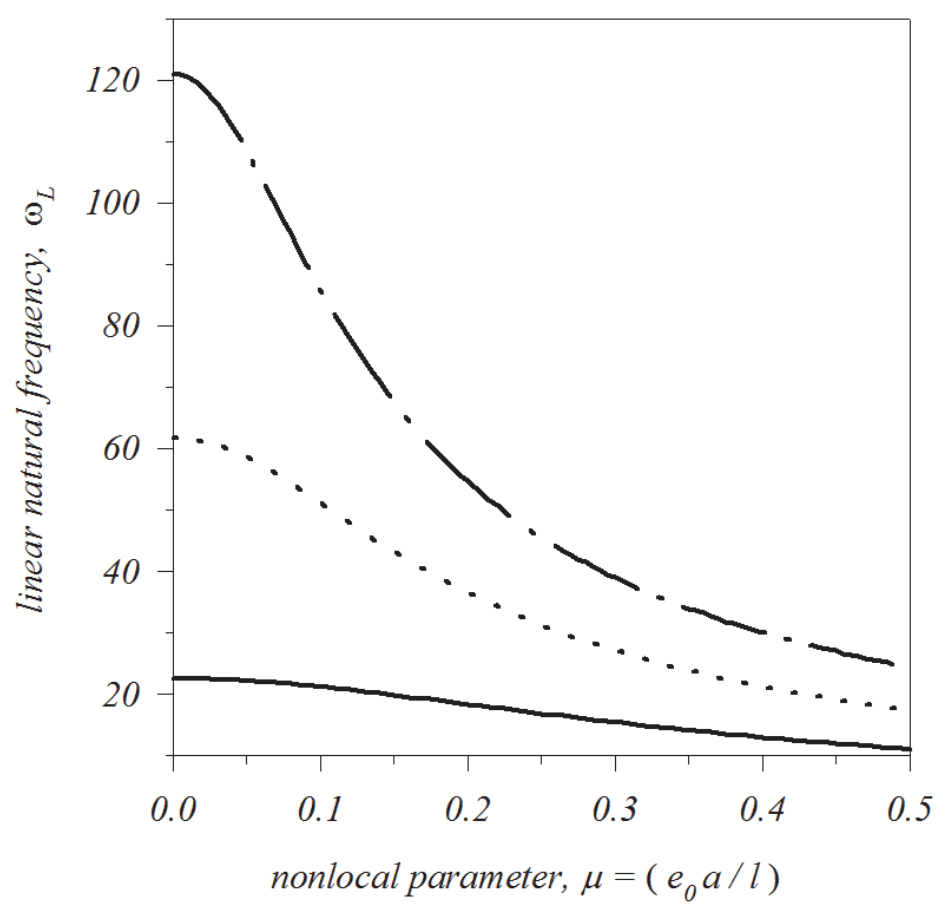

Figure 3. Variation of linear natural frequencies $\omega$ versus the nonlocal parameter $\mu$ of the first three modes, for the case $b=0$. st mode, 2 nd mode, 3 rd mode

$$
b=5
$$

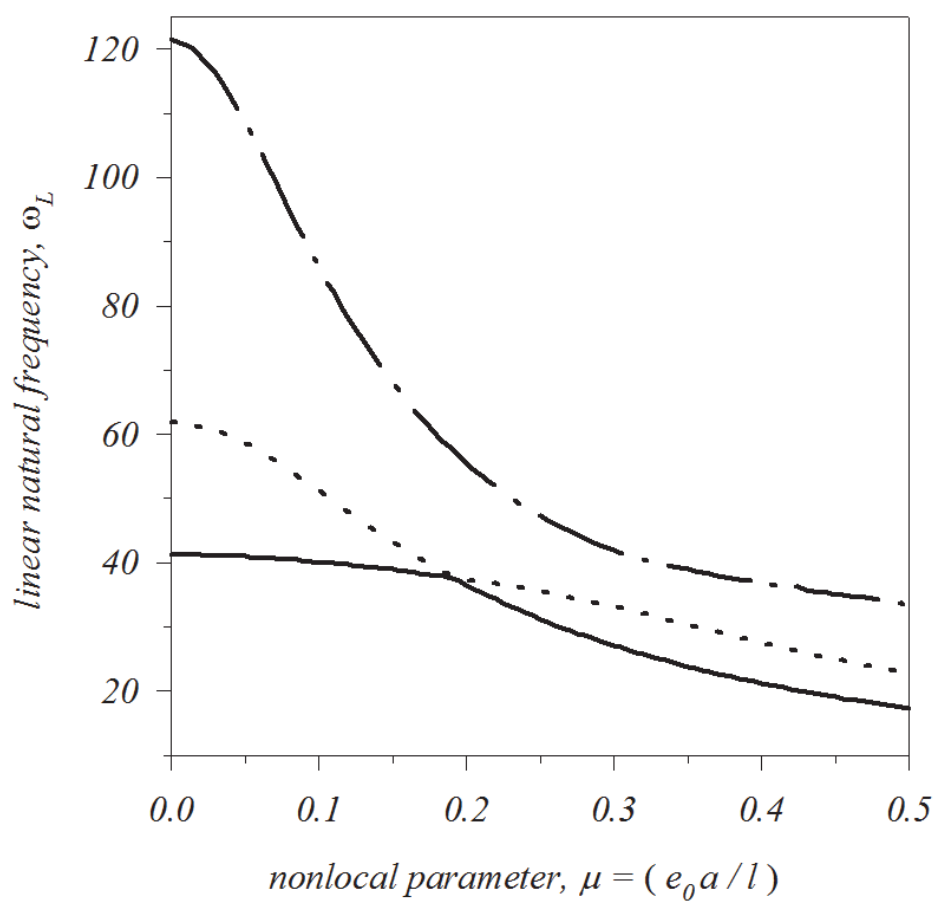

Figure 4. Same as in Figure (3), but for $b=5$ 


$$
b=10
$$

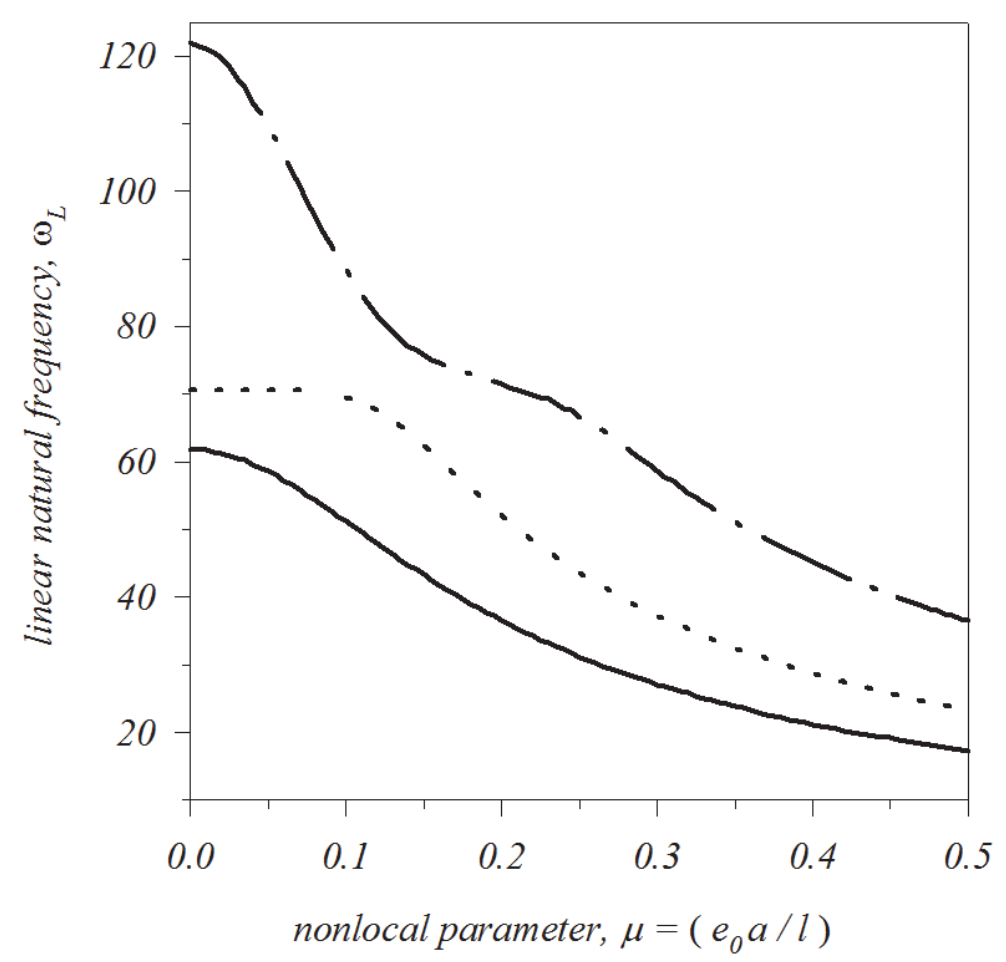

Figure 5. Same as in Figure (3), but for $b=10$

$$
b=15
$$

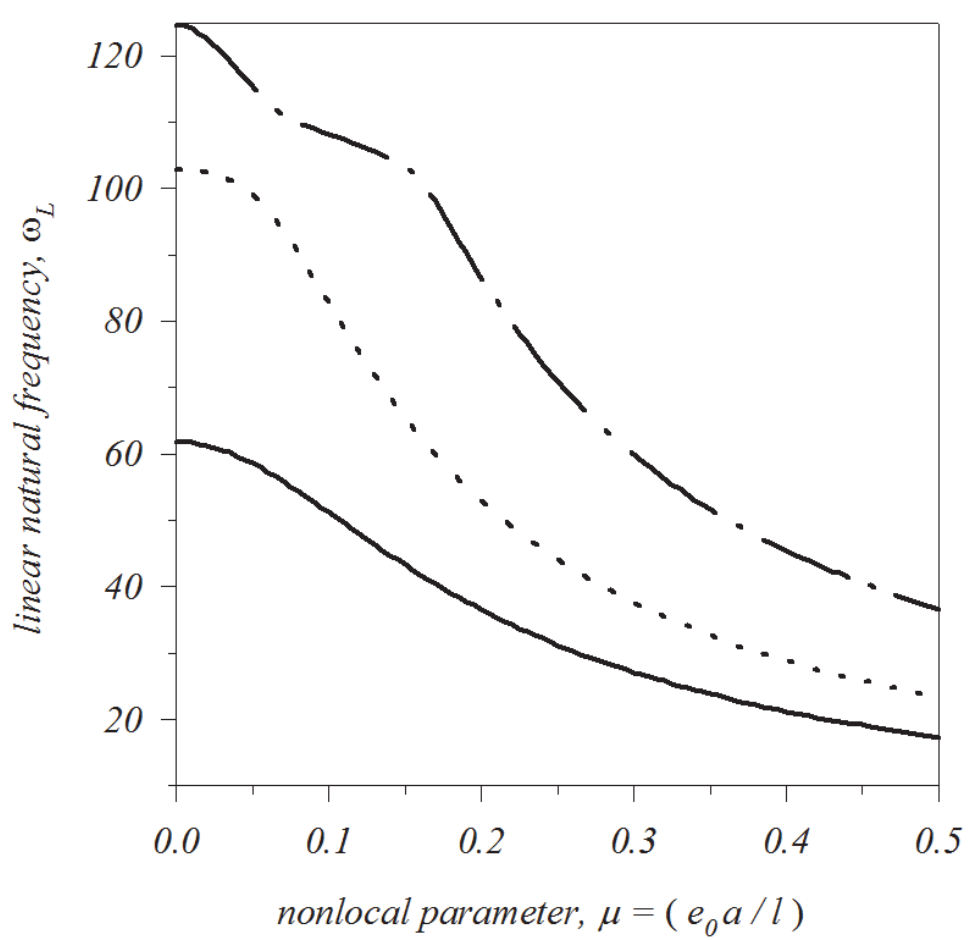

Figure 6. Same as in Figure (3), but for $b=15$ 
straight CNT, i.e. with no rise/imperfection and results for $\mu=0$, represents results for the local elasticity theory.

Figures (1 and 2) display the variation of the first four linear natural frequencies versus the CNT initial imperfection/rise for $\mu=0$ and $\mu=0.2$, respectively. It is well known that beams with initial rise, like the CNT under consideration, depending on system parameters, may exhibit a variety of complex dynamic behavior, e.g. frequency veering, mode localization, snap through, period doubling bifurcations and chaos (Ouakad \& Younis, 2011; Al-Qaisia \& Hamdan, 2010; 2009; 2013). These frequencies are obtained by solving the $4 \times 4$ coefficient matrix derived in section (3). It is clear that the veering occurs between the $1^{\text {st }}$ and $2^{\text {nd }}$ natural frequencies; at $b \approx 8.35$ as shown in the zoomed view in Figure (1) for $\mu=0$ and at $b \approx 5$ for $\mu=0.2$. Other veering zones between the $2^{\text {nd }}$ and $3^{\text {rd }}$ frequencies and between $3^{\text {rd }}$ and $4^{\text {th }}$ frequencies are also present for the two cases but at different values of initial rise. As shown in the figures and as indicated in (Al-Qaisia \& Hamdan, 2010), for dynamical systems with rise/imperfection, two of the system natural frequencies (usually the lowest ones) tend to approach each other as a system parameter is varied (rise in the present study). Afterwards, the two frequencies may veer away with high local curvature or intersect transversely "crossover". Also, previous studies have shown that the frequency veering can lead to mode localization (Al-Qaisia \& Hamdan, 2013), bifurcation instability (Mayoof \& Hawwa, 2009), and significant changes of the associated mode shape functions (Al-Qaisia \& Hamdan, 2013). From Figures (1 and 2), one can conclude that using the nonlocal elasticity theory leads to reduction in natural frequencies associated with decrease in the gaps between the first and second veering zones. For example, the $1^{\text {st }}$ and $2^{\text {nd }}$ veering zones took place at $b \approx 8.35$ and $b \approx 17$ for $\mu=0$ and at $b \approx 5$ and $b \approx 8$ for $\mu=0.2$, respectively.

Examples of the obtained natural frequencies for the first three modes, for a given CNT rise as the nonlocal effect $\mu=e_{0} a / l$ varies, are shown in Figures (3-6). For instance, as indicated in the figures, increasing the rise $b$ from 0 to 5 "Figures ( 3 and 4 )" will cause the $1^{\text {st }}$ natural frequency to approach the $2^{\text {nd }}$ one at a value of $\mu=0.195$, then they veer away as the value of $\mu$ increases. On the other hand, further increase in the rise/imperfection $b$, leads to initiation of another veering zone between the $2^{\text {nd }}$ and $3^{\text {rd }}$ natural frequencies, as demonstrated in Figures (5 and 6). Results presented for the linear natural frequencies, i.e. Figures (1-6), indicate that the variation of the natural frequency with the CNT initial rise is not linear as the nonlocal parameter is varied, and vice versa. This depends on how the effect of one parameter is dominant over the other. For example, increasing the nonlocal parameter $\mu$ leads to decrease in natural frequency and the "qualitative and quantitative" reduction depends on the initial rise value $b$, whether it is before, at, or after the veering zones, i.e. between the $1^{\text {st }}$ and $2^{\text {nd }}$ or between the $2^{\text {nd }}$ and $3^{\text {rd }}$ natural frequencies.

Results for the effect of the nonlocal parameter and initial rise on nonlinear natural frequencies of the CNT, of the first mode, are presented in Figures (7-10).

The non-dimensional natural frequencies are presented as a ratio that relates the nonlinear to linear natural frequency $\omega_{N L} / \omega_{L}$ and obtained from equation (47). As indicated in (Nayfeh \& Mook, 1979), the MMS method gives results with high accuracy even for large values of vibration amplitude $A$.

It can be seen from these results that the nonlinearities are not of fixed type; e.g. the system behavior can be of hardening or softening type depending on the selected values of the CNT parameters, i.e. initial rise $b$ and the nonlocal effect $\mu=e_{0} a / l$. By examining nonlinear equation (46), the quadratic nonlinearity depends on the initial rise and the cubic nonlinearity depends on mid-plane stretching. It is to be noted that for this type of nonlinear oscillators which is similar to the one studied thoroughly in (Al-Qaisia \& Hamdan, 2009; 2013), the behavior of the CNT, i.e. softening or hardening, depends on the quantity of $\left(\varepsilon_{3}-10 \varepsilon_{2}^{2} / 9\right)$ wither it is positive, zero, or negative.

(Nayfeh \& Mook, 1979) concluded that for this class of nonlinear oscillator, which describes the nonlinear vibration of the CNT given in equation (46), for $\left(\varepsilon_{3}-10 \varepsilon_{2}^{2} / 9\right)>0$, the oscillator response exhibits a hardening behavior, and a softening behavior if $\left(\varepsilon_{3}-10 \varepsilon_{2}^{2} / 9\right)<0$.

Accordingly, regardless of the sign of $\varepsilon_{2}$, the quadratic nonlinearity has a softening effect and the nonlinear oscillator given in (46) is of softening type unless $\varepsilon_{3}>10 \varepsilon_{2}^{2} / 9$. Also, when $\varepsilon_{3}=10 \varepsilon_{2}^{2} / 9$, the effects of the two nonlinearities cancel each other and consequently the behavior resembles that of the corresponding linear oscillator (Nayfeh \& Mook, 1979).

In Figure (7), the behavior of the CNT is of hardening type for all values of the nonlocal parameter $\mu$ and vibration amplitude $A$. Upon increasing the value of the rise $b$, Figure (8), the behavior is of softening type for all values of the nonlocal parameter $\mu$, but to a certain value of the vibration amplitude $A$. For example, for $\mu=0.15$, the nonlinear natural frequency ratio $\omega_{N L} / \omega_{L}$ decreases as the amplitude increases and 
becomes equals to zero at $A=0.5$. Other values of the nonlocal parameter $\mu$, exhibit the same trend but with different values of vibration amplitudes. These results should be considered when studying the forced vibration of the

$$
b=1, \mu=0,0.05,0.1,0.15,0.2
$$

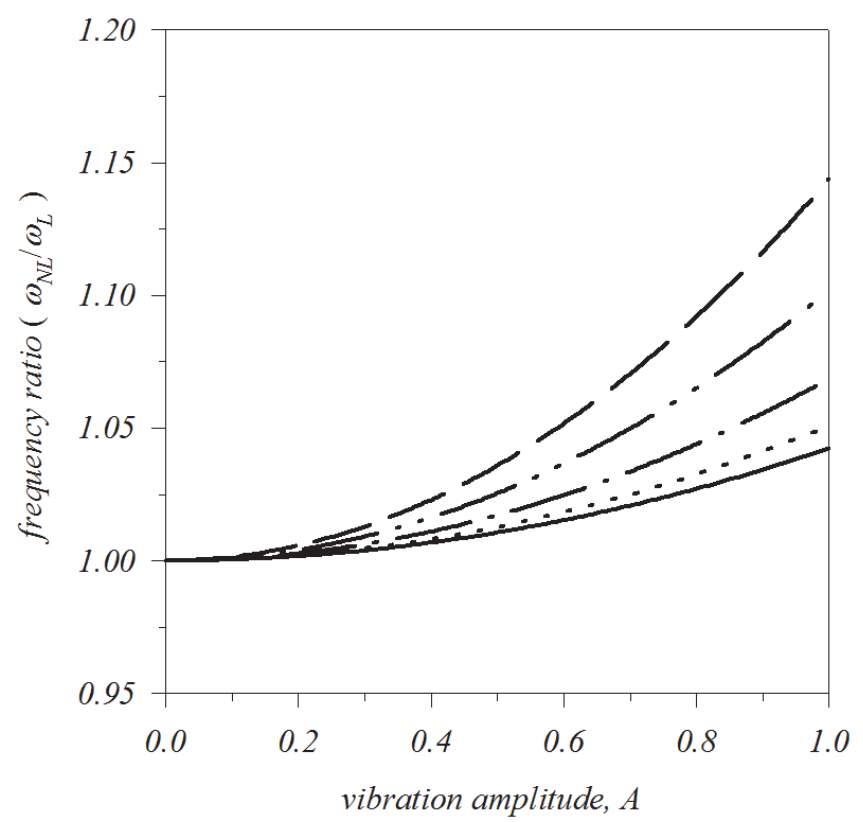

Figure 7. Nonlinear frequency ratio $\left(\omega_{N L} / \omega_{L}\right)$ versus the vibration amplitude $A$ of the fundamental frequency and $b=1$. $\mu=0$, $\mu=0.05, \ldots-\mu=0.10$, $\ldots \mu=0.15$

$$
\text { - - } \mu=0.20 \text {. }
$$

$$
b=4, \mu=0,0.05,0.1,0.15,0.2
$$

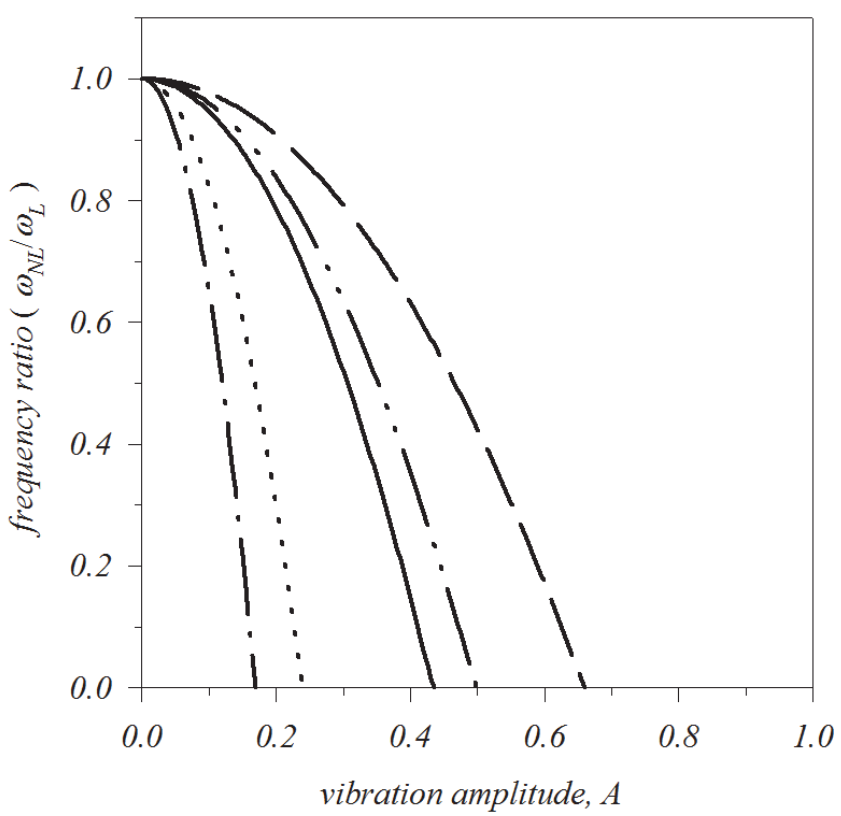

Figure 8. Same as in Figure (7) but for $b=4$ 


$$
b=7, \mu=0,0.05,0.1,0.15,0.2
$$

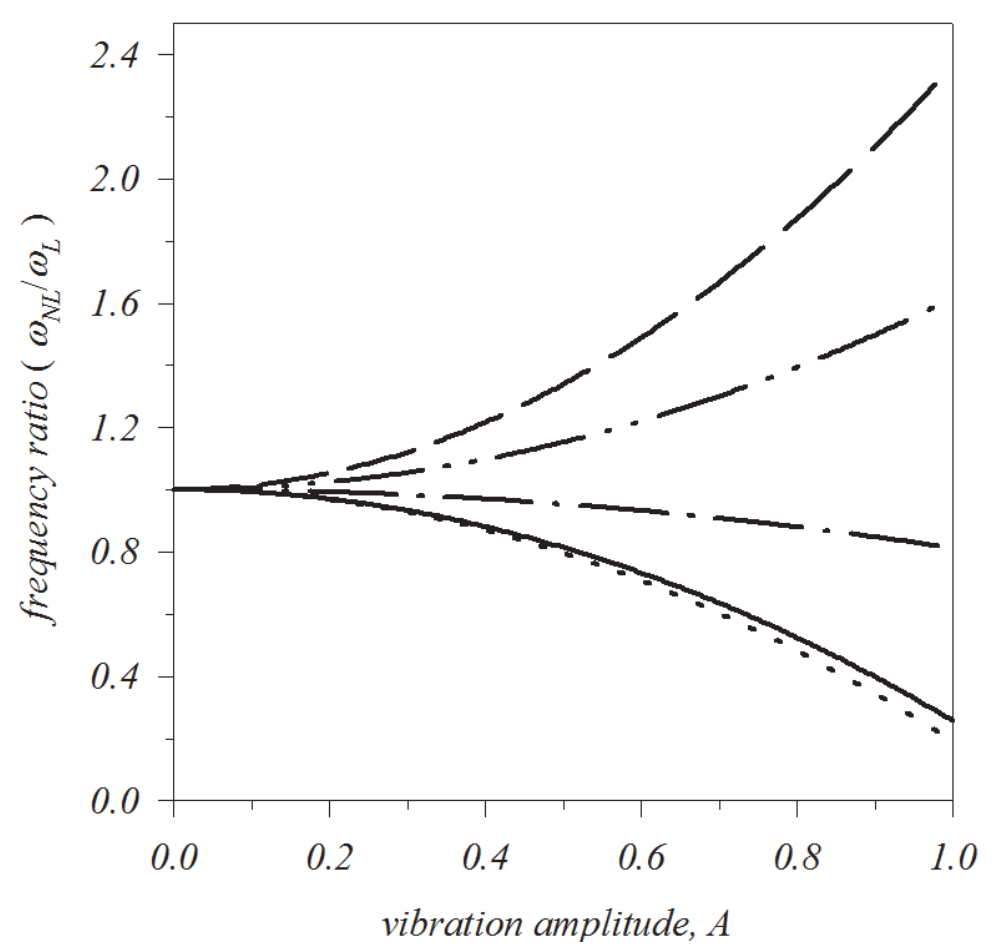

Figure 9. Same as in Figure (7) but for $b=7$

$$
b=10, \mu=0,0.05,0.1,0.15,0.2
$$

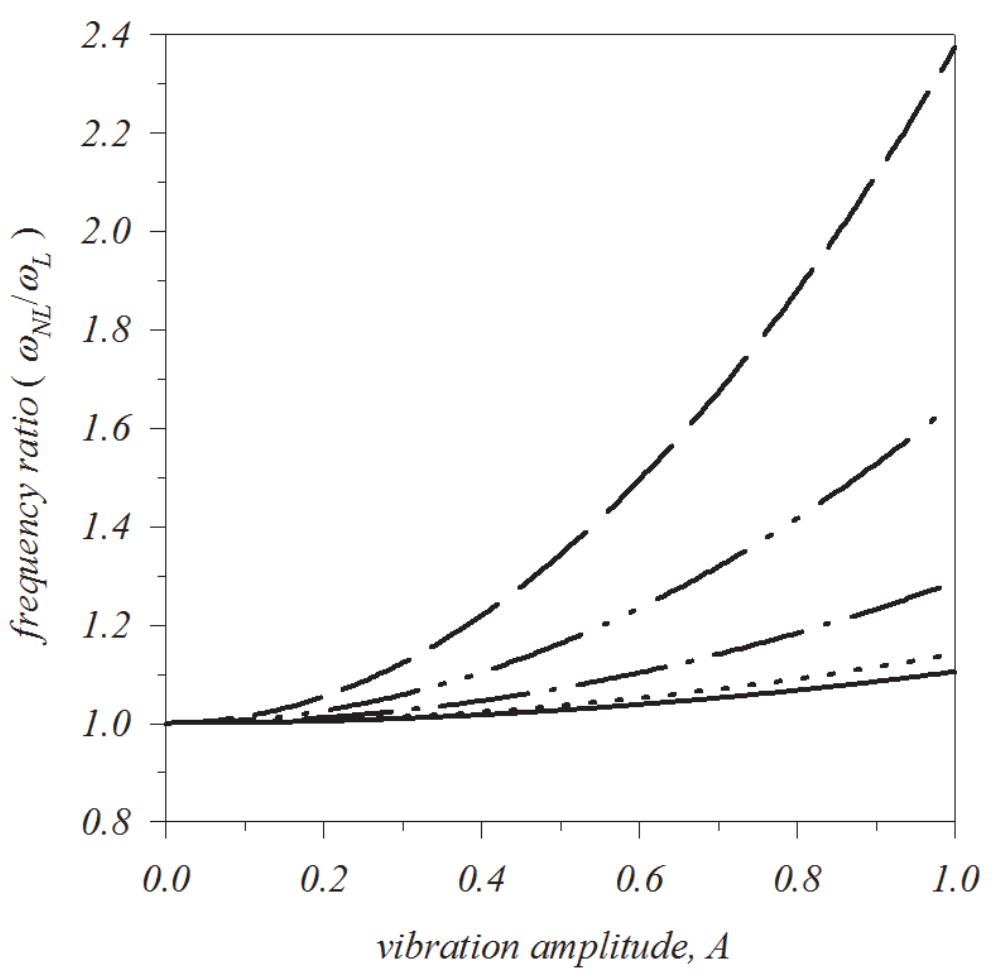

Figure 10. Same as in Figure (7) but for $b=10$. 


$$
b=5, \mu=0.2
$$

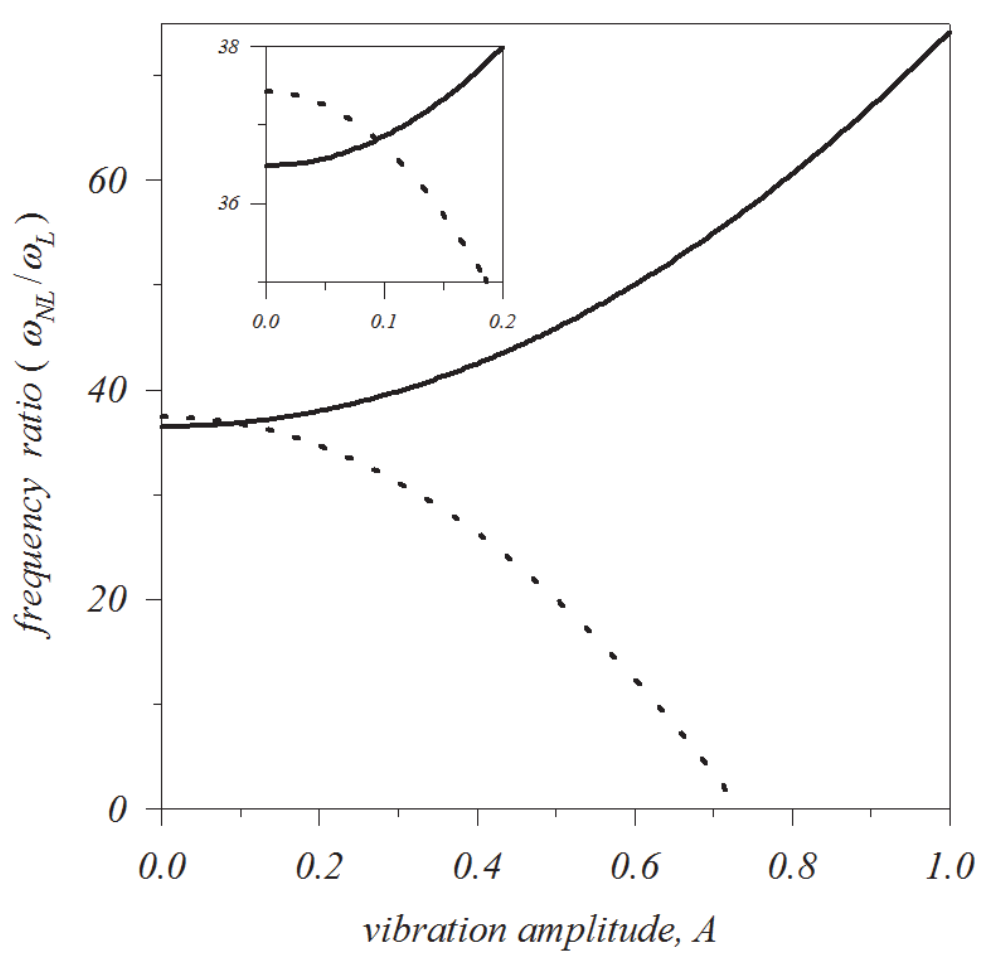

Figure 11. Nonlinear frequency ratio $\left(\left(\omega_{N L} / \omega_{L}\right)\right.$ versus the vibration amplitude $A$ of the $1^{\text {st }}$ and $2^{\text {nd }}$ modes for $b=5$ and $\mu=0.20$

$1^{\text {st }}$ mode,
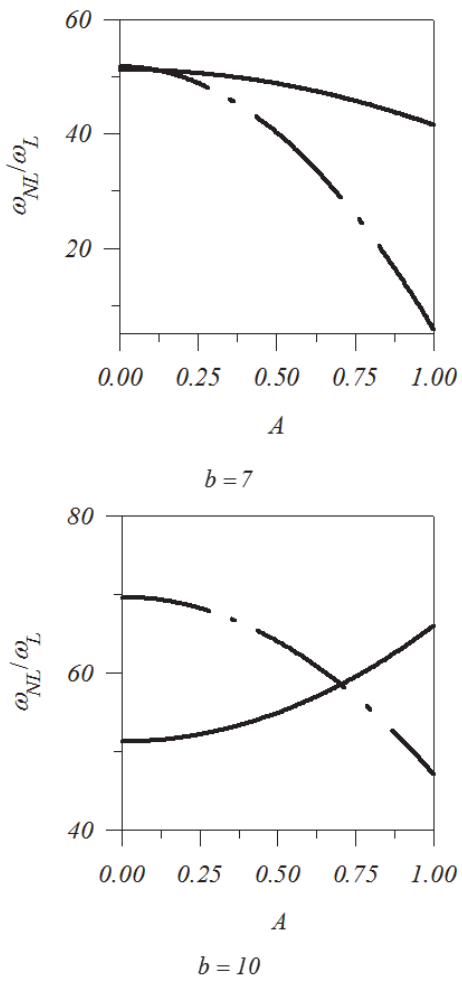

$2^{\text {nd }}$ mode.
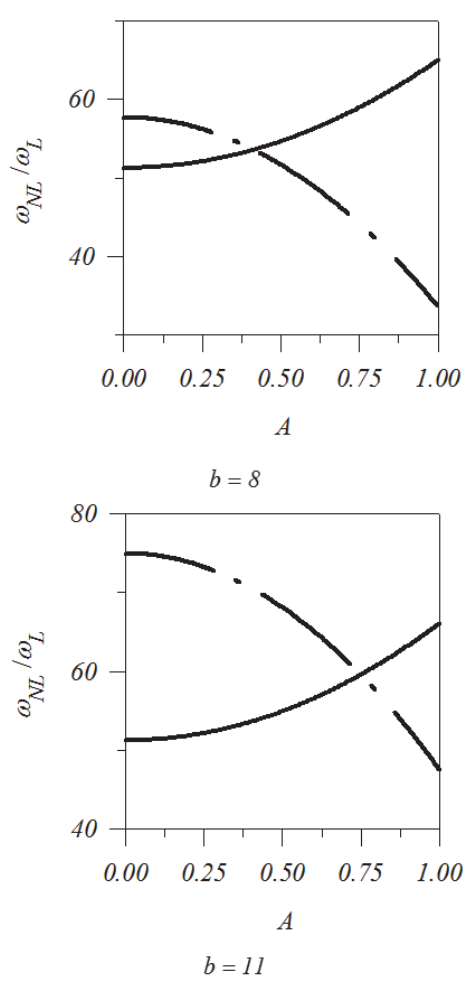

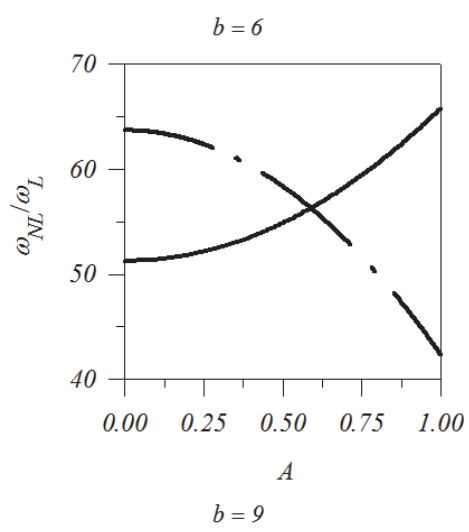

Figure 12. Nonlinear frequency ratio $\left(\left(\omega_{N L} / \omega_{L}\right)\right.$ versus the vibration amplitude $A$ of the $1^{\text {st }}$ and $2^{\text {nd }}$ modes near the $1^{\text {st }}$ veering zone for $\mu=0.1$ and different values of CNT rise $b$. $1^{\text {st }}$ mode, $2^{\text {nd }}$ mode 

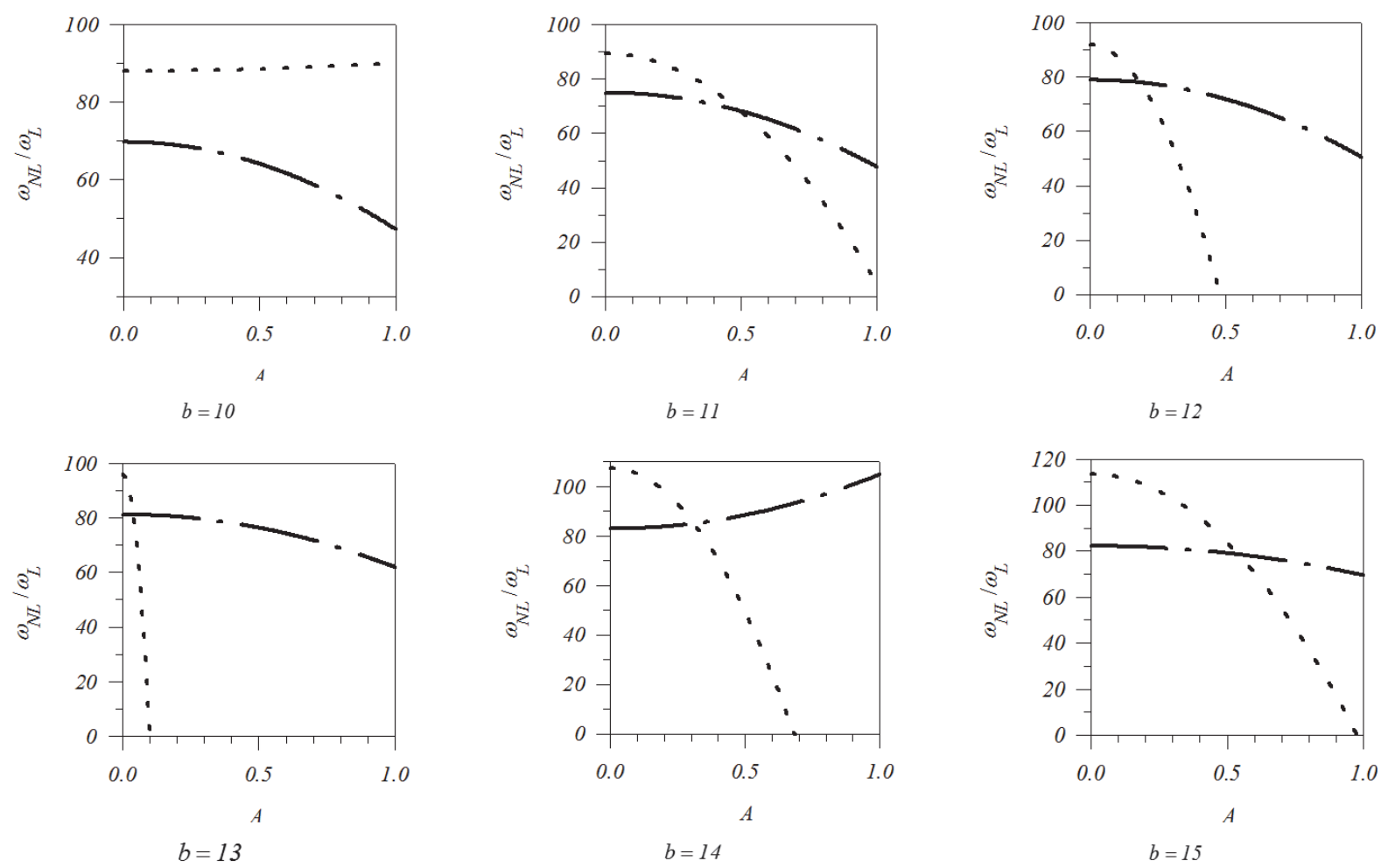

Figure 13. Nonlinear frequency ratio $\left(\left(\omega_{N L} / \omega_{L}\right)\right.$ versus the vibration amplitude $A$ of the $2^{\text {nd }}$ and $3^{\text {rd }}$ modes near the $2^{\text {nd }}$ veering zone for $\mu=0.1$ and different values of CNT rise $b$.

- . - $2^{\text {nd }}$ mode, ....... $3^{\text {rd }}$ mode

CNT, i.e. exciting the CNT with certain level and frequency that gives these responses, may lead to loss in its stability at which bifurcation and chaotic behavior take place.

Results obtained and presented in Figures (9 and 10) also indicate, depending on values of CNT initial rise $b$ and nonlocal parameter $\mu$, that the behavior may switch form softening to hardening depending on how the effect of the two parameters $b$ and $\mu$ compete with each other as the vibration amplitude increases.

The veering phenomenon, in general, may exhibit a complex dynamic behavior like; mode localization or cross over. As mentioned previously and as indicated in Figure (2), the veering took place between the $1^{\text {st }}$ and $2^{\text {nd }}$ natural frequencies at $b \approx 5$ for $\mu=0.2$. To examine this effect on the nonlinear behavior, results are obtained for the $1^{\text {st }}$ and $2^{\text {nd }}$ natural frequencies and presented in Figure (11). As shown in the figure, the behavior of the $1^{\text {st }}$ and $2^{\text {nd }}$ modes of vibration are of hardening and softening types, respectively. Here, the two modes intersect at an amplitude of $A=0.1$ " as shown in the zoomed view" and exhibit a cross over behavior. Again, exciting the CNT under these conditions, i.e. $b \approx 5$ and $\mu=0.2$, may lead to chaotic behavior or internal resonance between the two modes.

Finally, and to shed some light on how the cross over develops, results are obtained and presented in Figures (12 and 13) between the $1^{\text {st }}$ and $2^{\text {nd }}$ modes and between the $2^{\text {nd }}$ and $3^{\text {rd }}$ modes, respectively, but for $\mu=0.1$.

In Figure (12), the $1^{\text {st }}$ mode switches from softening to hardening and the $2^{\text {nd }}$ mode switches from hardening to softening as the CNT initial rise increases. Here, the nonlinear analysis matches with linear results presented in Figures ( 1 and 2), and the veering takes place at a given value of rise $b$ and nonlocal parameter $\mu$, but in the nonlinear analysis, in addition to veering phenomenon, the cross over becomes evident as the vibration amplitude increases. The same trend for the results is shown in Figure (13), but for the $2^{\text {nd }}$ and $3^{\text {rd }}$ modes of vibration. This behavior is due to the competence between the quadratic and cubic nonlinearities resulted from the effect of the CNT initial rise/imperfection with the nonlocal parameter as the vibration amplitude varies.

\section{Conclusions}

The nonlinear vibrational mathematical model for the single walled carbon nanotubes (SWCNT) was derived and developed based on the nonlocal elasticity theory and continuum mechanics. The CNT was modeled as 
Euler-Bernoulli beam with initial rise and clamped at both ends. The Hamilton's principle was used to derive the nonlinear integro-partial differential equation of motion and s single mode Galerkin's approach was used to discretize and reduce the equation to a uni-modal single temporal equation. The effect of CNT initial rise and nonlocal parameter were studied and investigated thoroughly and presented results for the analyses of the nonlinear free vibration of the CNT may exhibit a very complex dynamic behavior like; frequency veering and crossover instability, depending on the amplitude of the initial rise as well as the value of the nonlocal parameter. It was demonstrated that the nonlocal parameter, along with the initial imperfection, have a significant effect on the qualitative and quantitative linear and nonlinear natural frequencies.

Results obtained using the MMS method for different values of CNT initial rise and nonlocal parameter suggest that a more detailed analysis is required to study the qualitative behavior and to have a global picture on the forced vibration of the CNT nonlinear response governed by equation (46), near or at the frequency veering zones, which is currently under consideration.

\section{References}

Al-Qaisia, A., \& Hamdan M. (2009). Non-linear frequency veering in a beam resting on elastic foundation. Journal of Vibration and Control, 15, 1627-1647. https://doi.org/10.1177/1077546309103262

Al-Qaisia, A., \& Hamdan M. (2010). Primary resonance response of a beam with a differential edge settlement attached to an elastic foundation. Journal of Vibration and Control, 16, 853-877. https://doi.org/10.1177/1077546309339419

Al-Qaisia, A., \& Hamdan M. (2013). On nonlinear frequency veering and mode localization of a beam with geometric imperfection resting on elastic foundation. Journal of Sound and Vibration, 33, 4641-4655. https://doi.org/10.1016/j.jsv.2013.03.031

Arash, B., \& Wang, Q. (2012). A review on the application of nonlocal elastic models in modeling of carbon nanotubes and graphenes. Computational Materials Science, 51(2012), 303-313. https://doi.org/10.1016/j.commatsci.2011.07.040

Civalek, O., \& Demir, C. (2011). Bending analysis of microtubules using nonlocal Euler-Bernouli beam theory. Applied Mathematical Modelling, 35, 2053-2067. https://doi.org/10.1016/j.apm.2010.11.004

Eltaher, M., Emam, S., \& Mahmoud, F. (2012). Static and stability analysis of nonlocal functionally graded nanobeams. Composite Structures, 96, 82-88. https://doi.org/10.1016/j.compstruct.2012.09.030

Eringen, A., \& Edelen, D. (1972). On nonlocal elasticity. International Journal of Engineering Sciences, 10(3), 233-248. https://doi.org/10.1016/0020-7225(72)90039-0

Fang, B., Zhen, Y., Zhang, C., \& Tang, Y. (2013). Nonlinear vibration analysis of double-walled carbon nanotubes based on nonlocal theory. Applied Mathematical Modelling, 37, 1096-1107. https://doi.org/10.1016/j.apm.2012.03.032

Farshidianfar, A., \& Soltani, P. (2012). Nonlinear flow-induced vibration of a SWCNT with geometrical imperfection. Computational Materials $\quad$ Science, $\quad 53, \quad 105-116$. https://doi.org/10.1016/j.commatsci.2011.08.014

Harik, V. (2002). Mechanics of carbon nanotubes applicability of the continuum-beam models. Computational Materials Science, 24, 328-342. https://doi.org/10.1007/978-94-017-9263-9_2

Hawwa, M., \& Al-Qahtani, H. (2010). Nonlinear oscillations of a double-walled carbon nanotubes. Computational Materials Science, 48, 140-143. https://doi.org/10.1016/j.commatsci.2009.12.020

Kong, S., Zhou, S., Nie, Z., \& Wang, K. (2008). The size-dependent natural frequency of Bernouli-Euler micro-beams. International Journal of Engineering Science, 46, 427-437. https://doi.org/10.1016/j.ijengsci.2007.10.002

Lijima S. (1991). Helical microtubules of graphite carbon. Nature, 354(6348) 56-58. https://doi.org/10.1038/354056a0

Mayoof, F., \& Hawwa, M. (2009). Chaotic behavior of curved carbon nanotubes under harmonic excitation, Chaos. Solitons and Fractals, 42, 1860-1867. http://dx.doi.org/10.1016/j.chaos.2009.03.104

Mehdipour, I., \& Barari, A. (2012). Why the center-point of bridged carbon nanotubes length is the most mass sensitive location for mass attachment? Computational Materials Science, 55, 136-141. https://doi.org/10.1016/j.commatsci.2011.11.036 
Mehdipour, I., Barari, A., Kimiaefar, A., \& Domairry, G. (2102). Vibrational analysis of curved single-walled carbon nanotubes on a Pasternak elastic foundation. Advances in Engineering Software, 48, 1-5. https://doi.org/10.1016/j.advengsoft.2012.01.004

Mettler, E. (1962). Dynamic Buckling. In W. Flugge (Ed.), Handbook of Engineering Mechanics, (pp.62-1-11). New York, USA, McGraw-Hill.

Mustapha, K., \& Zhong, Z. (2012). Stability of single-walled carbon nanotubes and single-walled carbon nanocones under self-weight and axial tip force. International Journal of Engineering Science, 50, 268-278. https://doi.org/ 10.1016/j.ijengsci.2010.12.006

Nayfeh, A. H., \& Mook, D. T. (1979). Nonlinear Oscillations, New York, Wiley-Interscience. https://doi.org/f10.1002/9783527617586

Ouakad, H., \& Younis, M. (2011). Natural frequencies and mode shapes of initially curved carbon nanotubes resonators under electric excitation. Journal of Sound and Vibration, 330, 3182-3195. https://doi.org/10.1016/j.jsv.2010.12.029

Pantano, A., Boyce, M., \& Parks, D. (2003). Nonlinear structured mechanics based modeling of carbon nanotubes deformation. Physical Review letters, $91(145504), \quad 1-4$. https://doi.org/10.1103/PhysRevLett.91.145504

Papanikos, P., Nikolopoulos, D., \& Tserpes, K. (2008). Equivalent beams for carbon nanotubes. Computational Materials Science, 43, 345-352. https://doi.org/10.1016/j.commatsci.2007.12.010

Roque, C., Ferreira, A., \& Reddy, J. (2011). Analysis of Timoshenko nanobeams with a nonlocal formulation and meshless method. International Journal of Engineering Science, 49, 976-984. https://doi.org/10.1016/j.ijengsci.2011.05.010

Sazonova, V., Yaish, Y., Ustunel, H., Rounday, D., Arias, T., \& McEuen, P. (2004). A tunable carbon nanotubes electromechanical oscillator. Nature, 431, 284-287. https://doi.org/10.1038/nature02905

Thai, H. (2012). A nonlocal beam theory for bending, buckling, and vibration nanobeams. International Journal of Engineering Science, 52, 56-64. https://doi.org/10.1016/j.ijengsci.2011.11.011

Wang, Q., \& Liew, K. M. (2007). Application of nonlocal continuum mechanics static analysis of micro- and nano-structures. Physics letter A, 363, 236-242. https://doi.org/10.1016/j.physleta.2006.10.093

Wang, Q., \& Wang, C. M. (2007). The constitutive relation and small scale parameter of nonlocal continuum mechanics for modeling carbon nanotubes. Nanotechnology, 18, 075702 (4pp). https://doi.org/10.1088/0957-4484/18/7/075702

Xia, W., \& Wang, L. (2010). Vibration Characteristics of fluid-conveying carbon nanotubes with curved $\begin{array}{lllll}\text { longitudinal shape. } & \text { Computational }\end{array}$ https://doi.org/10.1016/j.commatsci.2010.04.030

Zhang, Y., Liu, G., \& Xie, X. (2005). Free transverse vibrations of double-walled carbon nanotubes using a theory of nonlocal elasticity. Physical Review B, 71(195404), 1-7. https://doi.org/10.1103/PhysRevB.71.195404

\section{Copyrights}

Copyright for this article is retained by the author(s), with first publication rights granted to the journal.

This is an open-access article distributed under the terms and conditions of the Creative Commons Attribution license (http://creativecommons.org/licenses/by/4.0/). 